U.Iowa 94-15

hep-lat/9409021

February 24, 1995

\title{
The Elusive Asymptotic Behavior of the High-Temperature Expansion of the Hierarchical Ising Model
}

\author{
Y. Meurice and G. Ordaz \\ Department of Physics and Astronomy, University \\ of Iowa, Iowa City, Iowa 52242, USA
}

\begin{abstract}
We present a differential formulation of the recursion formula of the hierarchical model which provides a recursive method of calculation for the high-temperature expansion. We calculate the first 30 coefficients of the high temperature expansion of the magnetic susceptibility of the Ising hierarchical model with 12 significant digits. We study the departure from the approximation which consists of identifying the coefficients with the values they would take if a $[0,1]$ Padé approximant were exact. We show that, when the order in the high-temperature expansion increases, the departure from this approximation grows more slowly than for nearest neighbor models. As a consequence, the value of the critical exponent $\gamma$ estimated using Padé approximants converges very slowly and the estimations using 30 coefficients have errors larger than 0.05. A (presumably much) larger number of coefficients is necessary to obtain the critical exponents with a precision comparable to the precision obtained for nearest neighbor models with less coefficients. We also discuss the possibility of constructing models where a $[0,1]$ Padé approximant would be exact.
\end{abstract}




\section{Motivations, Main Results and Notations}

The hierarchical mode ${ }^{[1]}$ is a model for which the renormalization group transformation ${ }^{[2]}$ reduces to a simple recursion formula. This recursion formula is a simple integral equation which has been studied in great detail..$^{[3]}$ More recently, interesting results concerning the analyticity and the location of the complex zeroes of the probability distribution associated with the infrared fixed point have been found. ${ }^{[4]}$ The hierarchical model has a free parameter $\epsilon$ which is used in the $\epsilon$-expansion. By adjusting this parameter, one can use the hierarchical model as an approximation ${ }^{[1-5]}$ to nearest neighbor models in various dimensions. In the case $D=3$, the hierarchical approximation yields values of the critical exponents which agree within a few percent with the best estimates for nearest neigbor models.

A difficult but interesting question is how to improve the hierarchical approximation while keeping control on the complexity of the renormalization group transformation. This question can be answered for gaussian models using grouptheoretical methods. ${ }^{[6]}$ However, the extension of these methods to interacting models is not straightforward because many more terms can be generated perturbatively. The main problem consists in identifying the most important perturbations which should be added to the hierarchical model in order to obtain a critical behavior more similar to nearest neighbor models. A possible indicator which could be used to achieve this goal is to calculate the effect of a perturbation on the hightemperature coefficients. Before evaluating the effects of these perturbations, it is necessary to develop efficient methods to calculate the high-temperature expansion of the (unperturbed) hierarchical model. Recently, analytical formula for the first four coefficients of the magnetic susceptibility were calculated for a Ising measure. ${ }^{[7]}$ The method used relies on high-temperature graphs. Due to the non-locality of the model, the terms appearing in the coefficients rapidly proliferate and require elaborate symbolic methods to be handled.

In this article, we present a recursive method which allows an efficient calculation of the high-temperature expansion of the hierarchical model. This method 
combines the renormalization group method whith the high-temperature expansion but does not rely on any graphical analysis. It is a simple differential version of the recursion relation (with an arbitrary rescaling of the spin variable). It essentially solves the problem of the high-temperature expansion provided that we keep the numerical stability and the volume effects under control. This method is explained in section 2 where we also calculate the first 30 coefficients of the high-temperature expansion of the magnetic susceptibility of the hierarchical Ising model in a large $\left(2^{60}\right.$ sites) but finite volume. Checking with analytical results and comparing different implementations of the differential recursion formula, we claim that for $\epsilon=1$, the method is numerically stable as far as 12 significant digits are concerned, and that within this precision, the first 30 coefficients have reached their infinite volume limit when $2^{60}$ sites are reached. We also briefly discuss the possible use of the differential formula to obtain analytical results concerning the high-temperature expansion.

In section 3, we study the departure from the approximation which consists of identifying the coefficients with the values they would take if the $[0,1]$ Padé approximant for the logarithmic derivative of the susceptibility were exact. The resulting approximate relation among the coefficients was suggested by a numerical study ${ }^{[8]}$ where we calculated (without any approximation) the magnetic susceptibility of the hierarchical Ising model with up to $2^{18}$ sites. In this study, we found that the numerical data can be fitted very precisely with a simple power law of the form $\left(1-\beta / \beta_{0}\right)^{-g}$ in the whole high-temperature region, i.e., for $\beta \in\left[0, \beta_{c}\right)$. The values of $g$ obtained from the numerical data or from the $[0,1]$ approximant, differ significantly from the best estimates of the values of the critical exponent $\gamma$ calculated $^{[3,9]}$ with the $\epsilon$-expansion. In the case of the numerical data, the most plausible explanation provided by a a renormalization group analysis ${ }^{[8]}$ is that this discrepancy is due to the limited size of the lattice used in these calculations. As we shall recall in the following section, if the lattice has $2^{n}$ sites, the recursion formula can only be used $n$ times, and $n=17$ does not seem to be enough to get rid of the irrelevant components of the measure. On the other hand, the fact that 
a $[0,1]$ approximant gives poor estimate is not surprising. What is surprising is how slowly the departure from this approximation grows when the order in the high-temperature expansion increases.

In section 4, we use Padé approximants to estimate $\gamma$ and $\beta_{c}$. We first discuss how the results depend on the volume. We show that when the volume is not too large - less or much less than $2^{20}$ sites for the orders considered here - the approximants can resolve a pair of complex conjugate roots approaching the real axis in the complex temperature plane. However, for larger volume, two real roots appear with - in a large majority of cases - only one root stabilizing near $\beta_{c}$. This stabilization toward the infinite volume value seems to occur with a precision comparable to the precision of the coefficients. We have checked this statement for the six approximants which can be calculated from the exact ${ }^{[7]}$ values of the first four coefficients at infinite volume. We concluded that the errors on the estimation of the critical quantities due to finite volume or round-off errors were very small compared to the size of the effects discussed below. More precisely, the 12 significant digits precision on the infinite volume coefficients are more than sufficient to discuss effects of order $10^{-3}$ in the critical quantities. The results for the 435 approximants that one can obtain from the coefficients up to order 30 are then presented. The estimates of $\beta_{c}$ are slightly too high for low order approximants but seem to converge reasonably well (i.e., small errors in the fourth significant digit in most of the cases) when the order becomes large enough. On the other hand, the estimates of $\gamma$ are significantly too high for low order approximants and evolve very slowly toward our best estimates. In order to give an idea, absolute errors of order 0.07 and 0.11 (respectively) are typical of the large order estimations for $\epsilon=1$ and 0 (respectively).

These large discrepancies can be understood in terms of the approximate relations discussed in section 3. For instance, the values of the critical exponent $\gamma$ extracted from a $[9,10]$ Padé is still midway in between the crude estimate given by the $[0,1]$ approximant (e.g., 1.52 for $\epsilon=1$ ) and the exact value $(1.30$, in the same case). This point is discussed at length in section 4 for $\epsilon=1$ and 0 . From 
these results, it appears that a (presumably much) larger number of coefficients is necessary to obtain the critical exponents of the hierarchical model with a precision comparable to these obtained ${ }^{[10]}$ for nearest neighbor model (i.e., a precision of 0.001 or better).

We also discuss (section 5 ) the possibility of constructing models where a $[0,1]$ Padé approximant would be exact. We first compare the approximate relations, for the hierarchical model and the nearest neighbor models when the dimensional parameter $D=4-\epsilon$ is varied. We show that the corrections to the Gaussian result (for which the approximation is exact) appear only at second order in the $1 / D-$ expansion ${ }^{[12]}$ for the hierarchical model while it appears in first order for nearest neighbor models. Unfortunately, the use of this expansion is obscured by a proliferation of zeroes and poles appearing in the expression of the coefficients when $D$ is promoted to a complex variable. ${ }^{[7]}$ We also mention the fact that supersymmetric models ${ }^{[13]}$ might be used as a guide to achieve this goal.

In the conclusions, we give various estimates of the number of coefficients necessary to calculate $\gamma$ with a reasonable precision. We discuss briefly the feasibility of the project and additional problems which could be considered in this context. We also compare with other recursive methods ${ }^{[11]}$ used to calculate the hightemperature expansion of the $3 D$ Ising model. This raises the following dilemma: on one hand, we have a much more simple and efficient method of calculation, on the other hand, we need much more coefficients to obtain a comparable information.

In order to give a self-contained presentation, we briefly remind our conventions concerning the hierarchical Ising model and its free parameter. Hierarchical models ${ }^{[1]}$ are specified by a non-local hamiltonian bilinear in the spin variables and a local measure of integration. In the following, we consider exclusively the case of an Ising measure, where the spin variables take only the values \pm 1 . The models considered here have $2^{n}$ sites. In the following, $n$ will always be used in this sense. We label the sites with $n$ indices $x_{n} \ldots . . x_{1}$, each index being 0 or 1 . The energy of 
a spin configuration reads

$$
H=-\frac{1}{2} \sum_{l=1}^{n}\left(\frac{c}{4}\right)^{l} \sum_{x_{n}, \ldots, x_{l+1}}\left(\sum_{x_{l}, \ldots, x_{1}} \sigma_{\left(x_{n}, \ldots, x_{1}\right)}\right)^{2}
$$

The motivations for this construction and the derivation of the recursion formula are reviewed in Ref. [8].

The model has a free parameter $c$ for which we shall use the parametrization

$$
c=2^{1-\frac{2}{D}}
$$

The parameter of the epsilon-expansion can be defined as

$$
\epsilon=4-D
$$

When $D \geq 4$, the model has a trivial continuum limit. ${ }^{[3]}$ When $D \leq 2$, the model does not have a phase transition at finite temperature. ${ }^{[1]}$ These two rigorous results can be understood heuristically in terms of the the self-intersection properties of the random walk associated with $H$, by noticing that the Haussdorff dimension of this random walk is $2 / D{ }^{[14]}$ From the point of view discussed at the beginning of the introduction, the most interesting region is $2 \leq D \leq 4$ (i.e., $1 \leq c \leq 2^{1 / 2}$ ), however, the model has a sensible infinite volume limit for a wider range of the free parameter, namely $0<D<\infty$ (i.e., $0<c<2$ ). We define the magnetic susceptibility per site as

$$
\chi_{n}(\beta)=\frac{1}{2^{n}}<\left(\sum_{x} \sigma_{x}\right)^{2}>_{n}
$$

The high-temperature expansion of this quantity reads

$$
\chi_{n}(\beta)=1+b_{1, n} \beta+b_{2, n} \beta^{2}+\ldots
$$




\section{A Recursive Method for the Calculation of the High-Temperature Expansion}

Due to the non-locality of the hierarchical model, the evaluation of the coefficients is rather tedious. We have used three types of techniques to calculate these coefficients. Two of them have been described at length in Ref. [7]. Up to order 4 , analytical calculations can be performed using algebraic methods ${ }^{[7]}$ and yield expressions where $D$ and $n$ are arbitrary. Substituting numerical values into these formulas provides very precise numerical comparisons with other numerical methods. We checked the validity of the analytical results by introducing a hightemperature expansion in the numerical method used in Ref. [8]. This numerical method is very reliable but rather slow and does not allow large $n$ calculations, because the computer time involved grows like $4^{n}$.

In order to perform large $n$ calculations, we have designed another method of calculation based on the Fourier transform version of the recursion relation. If we

call $\widehat{P}_{n}(k)$ the Fourier transform of the probability of the mean spin for $2^{n}$ sites, we obtain the recursion relation

$$
\widehat{P}_{n+1}(k)=C_{n+1} \exp \left(-\frac{1}{2} \beta\left(\frac{c}{4}\right)^{n+1} \frac{\partial^{2}}{\partial k^{2}}\right)\left(\widehat{P}_{n}(k)\right)^{2}
$$

where $C_{n+1}$ is adjusted in order to get $\widehat{P}_{n+1}(0)=1$ and the exponential of the second derivative is defined through the Taylor expansion. For the Ising model considered here, the initial function is

$$
\widehat{P}_{0}(k)=\cos (k)
$$

In this formulation, the magnetic susceptibility reads

$$
\chi_{n}(\beta)=-\left.\frac{1}{2^{n}} \frac{\partial^{2}}{\partial k^{2}} \widehat{P}_{n}(k)\right|_{k=0} .
$$

More generally, $\widehat{P}_{n}(k)$ generates the average values for the even powers of the mean 
spin:

$$
\widehat{P}_{n}(k)=\sum_{m=0}^{\infty} \frac{(-i k)^{2 m}}{2 m !}<\left(\sum_{x} \sigma_{x}\right)^{2 m}>_{n}
$$

Equation (2.1) is a differential formulation of the recursion formula. Unlike the original formulation, it does not involve any integrals and it can be reduced to a sequence of purely algebraic operations when expanded in powers of $\beta$ to a given order. In other words, (2.1) can be seen as a recursive definition of the high-temperature expansion of the average values of the even powers of the mean spin. This simple procedure completely solves the problem of the high-temperature expansion of the hierarchical model, provided that the operations can be performed within a reasonable amount of time and provided that we can reach the infinite volume limit with an acceptable numerical precision.

The computer time necessary to calculate the $m$ first coefficients grows like $m^{2} n$ since we only need to retain the first $2 m+2$ terms in the Taylor expansion of the functions introduced in the recursion formula. This is much better then the $4^{n}$ behavior reached by straightforward evaluation ${ }^{[7]}$ following the integration method of Ref. [8]. In order to give an idea concerning the absolute time scale, it takes a little less than two days, with Mathematica, to iterate 60 times Eq. (2.1) expanded up to order 30 .

Since the method requires many iterations, it is important to check the numerical stability of the algorithm. One potential numerical difficulty is the appearance of large numbers in (2.4), since one expects (see section 3 of Ref.[8]), that for large $n$,

$$
<\left(\sum_{x} \sigma_{x}\right)^{2 m}>_{n} \propto 2^{n m}
$$

This difficulty can be overcome either by replacing (2.1) by a recursion formula for the logarithm of $\widehat{P}_{n}$ divided by the number of sites, or by introducing an appropriate rescaling in (2.1). In the following, we only discuss the magnetic susceptibility and 
consequently, the second possibility is the most convenient one. In general, a rescaled version of (2.1) can be obtained by defining

$$
R_{\lambda, n}(k)=\widehat{P}_{n}\left(\frac{k}{\lambda^{n}}\right)
$$

In terms of this rescaled quantity, (2.1) becomes

$$
R_{\lambda, n+1}(k)=C_{n+1} \exp \left(-\frac{1}{2} \beta\left(\frac{c}{4} \lambda^{2}\right)^{n+1} \frac{\partial^{2}}{\partial k^{2}}\right)\left(R_{\lambda, n}\left(\frac{k}{\lambda}\right)\right)^{2}
$$

The large volume behavior shown in (2.5), can then be compensated by chosing $\lambda=\sqrt{2}$. This choice has been used for all the numerical results given hereafter. This modification improves significantly the precision of the calculation as discussed below. Note also that the renormalization group transformation is obtained by taking $\lambda=2 c^{-1 / 2}$ in $(2.7)$.

In the rest of this section and the next section, we discuss mostly the numerical results for $D=3$. Results for other values of $D$ will be discussed in sections 4 and 5 . Checking the numerical results obtained with the rescaled recursion formula (2.7) at $\lambda=\sqrt{2}$, for the first four coefficients, for all $n$ up to 60 , with the exact results at finite volume, ${ }^{[7]}$ we found errors of at most 1 in the 13 -th significant digit. This precision is almost twice better than the one obtained with the original formula (2.1). We have also compared slightly different numerical implementations for which the round-off errors should be different and found agreement at that level of precision for the 30 coefficients. We conclude that after 60 iterations, the numerical errors are at most in the 13-th significant digit.

The next question is to determine how close the coefficients calculated with $2^{60}$ sites are from the infinite volume ones. In order to get a first idea concerning the volume dependence of the coefficients, the results for $D=3$ and $n=10,20,30$ and 40 are given in Table 1. It is possible to compare the values of the first four 
coefficients with their infinite volume limit values: ${ }^{[7]}$

$$
\begin{aligned}
& b_{1}=1.242602432206561 \\
& b_{2}=1.280296258947416 \\
& b_{3}=1.249766644307651 \\
& b_{4}=1.171252787942138 .
\end{aligned}
$$

One sees that roughly, the agreement with the infinite volume limit improves by two digits each time that $n$ is increased by 10. This suggests that the volume dependence is exponentially suppressed. In order to check this possibility, we have plotted in Fig.1 the logarithm of $b_{m, 60}-b_{m, n}$ for $n<60$ and $m=1,5,10,15$, 20, 25, and 30. We have selected these few values of $m$ in order to keep the distinction among various values of $m$ visible on the figure. The trajectories for other $m$ fall approximately within the envelope delimited by the ones displayed. The figure makes clear the length of the plateau at low $n$ increases with $m$, in other words, higher order coefficients take a larger volume to stabilize (with exponential precision) near their infinite volume limit. After this, all the trajectories appear more or less parallel. The slope can be compared with the analytical results ${ }^{[7]}$ which show that the leading $n$-dependence of the first four coefficients comes from terms proportional to $\left(\frac{c}{2}\right)^{n}$. We remind that in the case considered here, $(D=3)$, $c=2^{1 / 3}$. The general agreement with this prediction is quite clear in Fig.1. In conclusion, Fig. 1 supports convincingly the possibility that the coefficients displayed reach their infinite volume limit with an exponential precision, and that up to the 30-th coefficient for $D=3$, the $n=60$ results give estimates of the infinite volume values which are accurate up to the 12-th significant digits (which is the limit of our numerical precision). The numerical results are given in Table 2 for $n=60$ and $D=3$ and also, for further reference, for $D=4$.

For comparison, we have also given in Table 1 the first 17 coefficients for the nearest neighbor model in three dimensions on a simple cubic lattice. These coefficients have been calculated from the tables given in Ref. [10]. Note that these 
tables are given for the expansion parameter $\tanh (\beta)$, and an additional expansion is necessary in order to obtain an expansion of the form of Eq. (2.5). In addition, we have rescaled the temperature of the nearest neighbor model in such a way that its first coefficient coincides with the one of the hierarchical model in the infinite volume limit. The large discrepancies indicate that the high-temperature coefficients should be sensitive to "non-hierarchical" perturbations.

Before closing this section, let us mention the possibility of using Eq. (2.7) to obtain analytical results. This equation relates the average value of the $2 m$-th power of the mean spin expanded to a certain order in $\beta$ to average values of larger $m$ and lower order in $\beta$. This suggest a bootstrap procedure which is presently under investigation. Note also that the $(c / 2)^{n}$ rule for the volume dependence appearing in Fig. 1, can be inferred from Eq. (2.7) with $\lambda=\sqrt{2}$ together with (2.5). Finally, let us mention that when $n$ goes to infinity, the recursion formula has a fixed point of the form $\exp \left(-\frac{1}{2} \chi k^{2}\right)$. The linearized transformation about this fixed point is presently under study.

\section{Approximate Relations among the High-Temperature Coefficients}

In a recent numerical calculation, ${ }^{[8]}$ we found that the numerical data for the susceptibility at $n=14,15$ and 16 can be fitted very precisely with a simple power law of the form $\left(1-\beta / \beta_{0}\right)^{-g}$ in the whole high-temperature region. As a consequence, it is possible to obtain a simple approximate formula for the hightemperature coefficients of the susceptibility in terms of $g$ and $\beta_{0}$ by comparing Eq. (2.5) and

$$
\left(1-\beta / \beta_{0}\right)^{-g}=1+\frac{g}{\beta_{0}}+\frac{g(g+1)}{2 \beta_{0}^{2}}+\ldots
$$

Approximate values for $g$ and $\beta_{0}$ are obtained by comparing the two first terms of 
Eqs. (3.1) and (2.5). Solving $\frac{g}{\beta_{0}}=b_{1, n}$ together with $\frac{g(g+1)}{2 \beta_{0}^{2}}=b_{2, n}$, we obtain

$$
\begin{aligned}
g & =\left(\frac{2 b_{2, n}}{b_{1, n}^{2}}-1\right)^{-1} \\
\beta_{0} & =\left(\frac{2 b_{2, n}}{b_{1, n}}-b_{1, n}\right)^{-1} .
\end{aligned}
$$

By comparing Eq. (2.5) with Eq. (3.1), and plugging the values of $\beta_{0}$ and $g$ given by Eq. (3.2), we obtain approximate values of the coefficients denoted $b_{m, n}^{a p p r}$. As an example, at third order we obtain

$$
b_{3, n}^{\text {appr. }}=\frac{g(g+1)(g+2)}{6 \beta_{0}^{3}} .
$$

Using Eq. (3.2), we then obtain

$$
b_{3, n}^{a p p r .}=\frac{b_{2, n}}{3}\left(\frac{4 b_{2, n}}{b_{1, n}}-b_{1, n}\right)
$$

Note that the approximate relations are homogeneous in the sense that at order $m$, all the terms have the form $b_{1, n}^{q} b_{2, n}^{p}$ with $2 p+q=m$.

In order to see how well these approximate relations are satisfied, we have calculated the difference between the exact and the approximate value of the coefficients denoted

$$
\Delta_{m, n}=b_{m, n}-b_{m, n}^{a p p r}
$$

Note that due to the homogeneity of the approximate relations mentioned above, the ratio $\Delta_{m, n} / b_{m, n}$ is invariant under a rescaling of the temperature. In particular, this implies that the rescaling of $\beta$ used in Table 1 for the nearest neighbor model has no effect on this ratio. The numerical values of this ratio for $D=3, m$ up to 30 , and $n=40$ and 60 are displayed in Table 3. The comparison between the results for the two values of $n$, shows that the volume dependence is not an issue for the discussion in the rest of this section. 
We can now compare the approximate relations for the hierarchical model and the nearest neighbor model. We see that in both cases the quality of the approximation deteriorates when $m$ increases. However, the rate are quite different: in the nearest neighbor case, $\Delta_{m, n} / b_{m, n}$ increases by approximately 0.04 each time $m$ is increased by 1 , while the corresponding increase is approximately 0.02 (and decreases with $m$ ) for the hierarchical model.

Equation (3.2), is exactly what we would have obtained from a $[0,1]$ Padé approximant for the logarithmic derivative of the susceptibility. In the infinite volume limit, we obtain $g=1.5189$ and $\beta_{0}=1.2224$. The value of $g$ is significantly larger than the value of the critical exponent $\gamma$ obtained ${ }^{[9]}$ in the $\epsilon$-expansion, namely 1.300. A value close to 1.30 has also been obtained with independent methods by Bleher. ${ }^{[3]}$ The value of $\beta_{0}$ is also significantly larger than our best estimate $^{[8]} \beta_{c}=1.179$. It is not a surprise that a $[0,1]$ Padé approximant provides bad estimates of the critical quantities. What is a surprise is how slowly the hightemperature series of the hierarchical model departs from this poor approximation when the order $m$ increases. This slower rate of departure implies that a number of coefficients much larger than in the nearest neighbor case, is necessary in order to estimate the critical quantities with a comparable precision.

We suspect that there exists a definite relationship between the quality of the estimates made from finite $n$ and infinite $m$ (as done in Ref.[8]) and those made from finite $m$ and infinite (or sufficiently large) $n$ (as done here). This remark is motivated by the observation that the values of $\gamma$ obtained at $n=16$ (e.g., 1.47 for $D=3$ ) are close to those obtained from low order Padé approximants (see next section). The discrepancy of the finite volume fits with the $\epsilon$-expansion was attributed ${ }^{[8]}$ to the fact that for $n=16$, for instance, we can only use the recursion formula 16 times, while a larger number seems necessary to get rid of the irrelevant components of the measure. This statement has a counterpart for the high-temperature expansion which is roughly the following: the coefficients which at a finite $n$, have reached their infinite value with an acceptable precision, provide estimates of the critical exponents consistent with the finite $n$ data. Since a direct 
calculation of the susceptibility at $n=30$ or 40 seems excluded with the exact method used in Ref. [8], we cannot sharpen further the statement made above. However, the $n$-dependence of the numerical estimates shown in Figs. 11 and 12 of ref. [8], the regularity of the length of the shoulders of Fig. 1 of this article and the results shown below suggest that a more quantitative study could be made in the future, possibly using the Eq. (2.7).

\section{Estimation of $\beta_{c}$ and $\gamma$ Using Padé Approximants}

In this section, we discuss the estimation of $\beta_{c}$ and $\gamma$, using $[L, M]$ Padé approximants with $L+M+1 \leq 30$. In the following, we call $L+M+1$ the order of the approximant. Since our calculation of the coefficients has been done at large but finite volume, we will first address the question of the volume dependence of the results obtained. As well-known, at finite volume, a singularity of the susceptibility on the real temperature axis is impossible. However, when the volume increases, a pair of complex conjugate singularities can approach arbitrarily closely the real axis. As we now proceed to explain, the Padé approximants sense this approach of the real axis, but with a finite resolution.

We have studied a large set of approximants using the finite volume coefficients and followed the motion of the roots of the denominator when the volume is increased. We have observed the following patterns in a large majority of cases. For $n$ below a number which is usually between 10 and 15, the roots move by large steps, usually of the same order as the change in the coefficients. During this preliminary motion, a pair of roots "pinches" the real axis and two clearly distinct real roots appear. This situation is illustrated in Fig. 3. One of these two real roots almost immediately stabilizes near $\beta_{c}$ (1.179 in this case), while the other makes a few erratic steps before stabilizing (more slowly) near another real value clearly distinct from $\beta_{c}$. Fig. 4 illustrates the small size motion of all the roots in

the complex plane for $n$ between 17 and 60. All the roots are well separated and for the roots labeled $1,3,4,5$, and 6 the changes are barely perceptible. We have 
skipped $n=14,15$ and 16, because the root labeled as 2 in Fig. 3 makes sudden changes during these 3 steps. Comparing with other cases, it appeared clearly that the value of $n$ for which the pinching of the real axis occurs increases with the order $(L+M+1)$ of the approximant. In other words, it appears that the Padé approximants of a given order can only resolve a pair of complex conjugate roots when their distance to the real axis is not less than a certain value (which decreases with the order of the approximant).

In most of the cases, the roots are widely separated for $n>20$. However, there are a few exceptional cases where two real roots are very close to $\beta_{c}$, for instance the $[13,12]$ and the $[12,13]$ approximants for $D=3$, and also $[10,11]$ and $[11,12]$ for $D=4$. In these special cases, the residues at both poles are very different from the residues at the pole near $\beta_{c}$ for most approximants of comparable order.

A closer look at the numbers indicates that the change in the location of the pole near 1.18 and the change (due to the volume) in the value of the residue at the pole decrease exponentially when the volume is increased. In the case of approximants requiring 4 coefficients or less, we can indeed obtain a precise comparison between the results at $n=60$ and $n=\infty$ using the infinite volume limit values of Eq. (2.8). We found that for the six approximants with $L+M+1 \leq 4$ the difference between the $n=60$ and $n=\infty$ appears at worse in the 11-th significant digit for both $\beta_{c}$ and $\gamma$. We also made independent random changes of order $10^{-4}$ in the values of a few coefficients and did not observe any instability in the estimations. Our general conclusion concerning the volume dependence of the estimations obtained from Padé approximants calculated with coefficients corresponding to models with $2^{60}$ sites, is that the results obtained approximate the infinite volume results with a precision which is far beyond what would be required to discuss effects of the order of $10^{-3}$ in the critical quantities.

We can now present the numerical results. In Table 4 and 5, we give the values of $\beta_{c}$ and $\gamma$ obtained from the $[j+k, j]$ approximants for $1 \leq j \leq 14, k= \pm 1,0$ and $D=3$ and 4 . In both cases, we have used the coefficients for $n=60$. We can now 
compare these results with our best estimates. In the case $D=3$, one observes a very slow (compared to nearest neighbor models) progression toward $\beta_{c}=1.179$ and $^{[3,9]} \gamma=1.300$ when the order of the approximants is increased. In the case $D=4$, a similar progression toward $\beta_{c}=0.665$ and the trivial value $\gamma=1$.

In order to give a more complete idea concerning the results obtained from Padé approximants, we give in Fig.5, the distribution of of values $\gamma$ obtained from the 435 different approximants of order less than 30 for $D=3$. Less than one half of one percent of the data fell outside of the figure. The figure shows separately the distribution for $L+M \leq 10,10<L+M \leq 20$ and $20<L+M<30$. The figure makes clear that the distribution gets more peaked and that the average decreases when the order of the approximants increases. The average for the distribution with $20<L+M<30$ is 1.382 which is still far away from 1.300. Note also that the number of approximants giving a value of $\gamma$ near 1.30 is non-negligible for $L+M<10$, however, this feature clearly fades away when the order increases.

\section{Are There Models for which the Approximate Relations are Exact?}

Up to now, the approximate relations discussed in section 3 have had a rather unpleasant effect: we need to calculate many coefficients in order to get any reliable result. However, the situation would be opposite if these relations were exact or if the departure could be estimated very precisely. It is thus tempting to try to construct models where, for instance, the $[0,1]$ Padé approximant would be exact.

A modest step in this direction consists in considering the $D$-dependence of the approximate relations studied in the special case $D=3$ in section 3 , and compare with the nearest neighbor models. In the rest of this section, we consider the infinite volume limit and the reference to $n$ disappears. In Fig. 2, we have plotted, $\Delta_{m} / b_{m}$ for $m=3$ and 4 as a function of $D$ using the exact analytical results of Ref. [7]. We see that both quantities decrease when $D$ becomes large and vanish for special values of $D$ between 1 and 2. A more detailed graph would 
show that $\Delta_{3}$ vanishes near $D=1.14$ and $\Delta_{4}$ near $D=1.66$. It is not clear that we can learn anything interesting from the behavior of the coefficients in this low $D$ region. On the other hand, the fact that the approximation becomes better for large $D$ is due to the fact that, as for the nearest neighbor models ${ }^{[12]}$, the corrections to the Gaussian result $b_{m} / b_{1}^{m}=1$ vanish. Note that the approximation discussed in section 3 is more general than the Gaussian approximation, since it allows $b_{2}$ to be distinct from $b_{1}^{2}$, however it is obviously exact in the gaussian approximation.

We first consider the nearest neighbor case. After an elementary calculation and an appropriate expansion of $\tanh (\beta)$, we obtain that for the Ising models with nearest neighbor interactions on an hypercubic lattice of dimension $D: b_{1}=2 D$, $b_{2}=-2 D+4 D^{2}, b_{3}=\frac{4 D}{3}-8 D^{2}+8 D^{3}, b_{4}=\frac{10 D}{3}+\frac{16 D^{2}}{3}-24 D^{3}+16 D^{4}$.

Expanding $\Delta_{m} / b_{m}$ in $1 / D$, we obtain:

$$
\left(\Delta_{3} / b_{3}\right)=\frac{1}{6 D}-\frac{1}{36 D^{3}}-\frac{1}{36 D^{4}}-\frac{5}{216 D^{5}}+\mathrm{O}\left(\frac{1}{D}\right)^{6}
$$

and

$$
\left(\Delta_{4} / b_{4}\right)=\frac{5}{12 D}-\frac{1}{4 D^{2}}-\frac{1}{18 D^{3}}-\frac{25}{288 D^{4}}-\frac{103}{1728 D^{5}}+\mathrm{O}\left(\frac{1}{D}\right)^{6}
$$

We can now compare with a similar expansion for the hierarchical model. Using the results of section 6 of Ref. [7], we obtain

$$
\left(\Delta_{3} / b_{3}\right)=\frac{8 \log (2)^{2}}{3 D^{2}}-\frac{512 \log (2)^{3}}{9 D^{3}}+\frac{2488 \log (2)^{4}}{3 D^{4}}-\frac{266432 \log (2)^{5}}{27 D^{5}}+O\left(\frac{1}{D}\right)^{6}
$$

and

$$
\left(\Delta_{4} / b_{4}\right)=\frac{20 \log (2)^{2}}{3 D^{2}}-\frac{1096 \log (2)^{3}}{9 D^{3}}+\frac{80084 \log (2)^{4}}{63 D^{4}}-\frac{2764360 \log (2)^{5}}{441 D^{5}}+O\left(\frac{1}{\mathrm{D}}\right)^{6}
$$

It is clear that the corrections to the Gaussian result appear at an higher order $\left((1 / D)^{2}\right)$ for the hierarchical model than for the nearest neighbor model. However 
a direct comparison of the two expansions does not shed any new light on Table 3. The reason being that for $D=3$, the series given in Eqs. (5.3-4) diverge. The origin of these divergences can be found from the zeroes and poles of the coefficients in the complex 1/D-plane (see Ref. [7] for more detail). For instance, in the case of Eq. (5.3), we found a pair of poles at $(1 / D)=-0.15608 \pm i 0.042037$ and then at $(1 / D)=0.25$ etc.... A complete description seems to be of little interest, since it does not suggest any way to improve the approximation.

We shall end this section with a speculative note. Supersymmetric gauge theories provide non-trivial examples where the dependence of some Green's functions on the mass is expected to be a simple power law, ${ }^{[13]}$ which is somehow the same thing as saying that Eq. (3.1) is exact. It is clearly difficult to compare these supersymmetric theories, which as far as we know do not have a satisfactory lattice regularization, with the spin models considered here. However, the non-locality of the hierarchical model suggests that it is an "effective" theory obtained after integrating over some other local variables. Indeed, a concrete example has been given in Ref. [15], where the hierarchical model was reformulated as a local theory with additional spin variables integrated with a Gaussian measure. It thus conceivable that one could find a reformulation of the hierarchical model where approximate symmetries playing a role similar to the supersymmetries, could be more manifest.

\section{Conclusions}

We have presented a recursive method of calculation which allows an efficient calculation of the high-temperature expansion of the hierarchical Ising model. The number of coefficients calculated is larger than what seems achievable ${ }^{[1011]}$ in the case of nearest neighbor models. However, our errors on the critical exponent $\gamma$ range between 0.05 and 0.1 and are much larger than the typical precision (0.001) obtained for nearest neighbor models with less than 20 coefficients. We have carefully considered the errors due to the fact that we used a large but finite volume to 
calculate the coefficients and concluded that these errors were much smaller than the effects described above.

We have identified the reason of this slow approach of the asymptotic regime by comparing the departure from the approximation corresponding to a $[0,1]$ Padé approximant. The last two columns of Table 3 indicate that at least 50 coefficients will be necessary in order to get a precision of 0.001 for $\gamma$. However, Tables 4 and 5 seem to leave open the possibility that a much larger number might be necessary. With the numerical method used in this paper, it would probably take a year to calculate 400 coefficients. We are presently working on an optimized version ${ }^{[16]}$ of the existing program which would allow us to achieve this goal in a shorter time.

Note that recursive methods have been found ${ }^{[11]}$ to calculate the high temperature expansion of the $3 D$ Ising model with nearest neighbor interactions. Their recursive step consists of adding one spin and the calculation requires large parallel computers to calculate 24 coefficients. Our recursive step consists of putting in contact two identical systems of size $2^{n}$ in order to get a system of size $2^{n+1}$ and 24 coefficients can be obtained within less than two days with a PC. On the other hand, with 24 coefficients, we obtain estimates which are less precise by one order of magnitude. We would like to check weather or not the following possibility occurs: our recursive algorithm would require a logarithmically smaller time of computation, but at the same time require an exponentially larger number of coefficients in order to reach a given precision.

Having at hand a large number of coefficients for the high-temperature expansion would allow precise comparison with the $\epsilon$-expansion for which a large number of coefficients is available. ${ }^{[9]}$ In the case of nearest neighbor models, discrepancies have been observed between these two methods. ${ }^{[17]}$ However, several authors ${ }^{[18]}$ have shown that these discrepancies can be removed by an appropriate treatment of the confluent singularities. For the hierarchical model, this is a completely open question. It is also possible that the simplicity of Eqs. (2.6-8) would allow to obtain analytical results concerning the asymptotic behavior of the high-temperature 
expansion.

Acknowledgements: One of us (Y.M.) would to thank the the theory group of Brookhaven National Laboratory and the Institut de Physique Théorique at Louvainla-Neuve for their hospitality while part of this work was completed.

\section{REFERENCES}

1. F. Dyson, Comm. Math. Phys. 12 (1969) 91 ; G. Baker, Phys. Rev. B 5 (1972) 2622 .

2. K. Wilson, Phys. Rev. B 4 (1971) 3185 ; K. Wilson and J. Kogut, Phys. Rep. 12 (1974) 75 .

3. P. Bleher and Y. Sinai, Comm. Math. Phys. 45 (1975) 247 ; P. Collet and J. P. Eckmann,Comm. Math. Phys. 55 (1977) 67 and Lecture Notes in Physics 74 (1978) .

4. H. Koch and P. Wittwer, Comm. Math. Phys. 106 (1986) 495 , 138 (1991) $537, \mathbf{1 6 4}(1994) 627$.

5. K. Gawedzki and A. Kupiainen, Les Houches 1985, K. Osterwalder and R. Stora, Editors

6. Y. Meurice, in Proceedings of the International Europhysics Conference on High Energy Physics, p. 89, Eds. J. Carr and M. Perottet, Editions Frontières, 1994.

7. Y. Meurice, Jour. Math. Phys. 36 (1995) 000 (in press), U. of Iowa preprint 94-14 hep-lat 9408021.

8. Y. Meurice, G. Ordaz and V.G.J. Rodgers, Jour. Stat. Phys. 77 (1994) 607 .

9. P. Collet, J.-P. Eckmann, and B. Hirsbrunner, Phys. Lett. 71B, 385 (1977).

10. D. S. Gaunt and A. J. Guttmann, in Phase Transitions and Critical Phenomena, (C. Domb and M. S. Green, eds.) Academic Press 1974. 
11. G. Bhanot, M. Creutz, U. Glasser and K. Schilling, Phys. Rev. B 49, 12909 (1994) .

12. M. Fisher and D. Gaunt Phys. Rev. 133A (1964) 224 .

13. G. Veneziano, Phys. Lett. B124 (1983) 357 ; for more references see: D. Amati, K. Konishi, Y. Meurice, G. C. Rossi and G. Veneziano, Phys. Reports 162 (1988) 169 .

14. Y.Meurice, Phys. Lett. 265B (1991) 377 ; J.L.Lucio and Y. Meurice, Mod. Phys. Lett. 6 (1991) 1199.

15. Y. Meurice, Mod. Phys. Lett. 7 (1992) 3331.

16. Y. Meurice, G. Ordaz and V. G. J. Rodgers, work in progress.

17. There is a large amount of literature on this subject. References can be found in Phase Transitions, Cargese 1980, M. Levy, J.C. Le Guillou and J. Zinn-Justin Editors, (Plenum Press, New York, 1982).

18. Z. Roskies Phys. Rev. B 24 (1981) 5305 ; J. Adler, M. Moshe and V. Privman Phys. Rev. B 26 (1982) 3958 ; G. Nickel and M. Dixon Phys. Rev. B 26 (1982) 3965 . 


\section{FIGURE CAPTIONS}

Fig.1: The logarithm of the difference between the largest volume value $b_{m, 60}$ and $b_{m, n}$ for $n<60$ is plotted for $m=1,5,10,15,20,25$ and 30 . The solid line is the the analytical slope for low $m$. The value of $D$ is 3 .

Fig.2: The difference between the exact and approximate values of the third and fourth coefficient divided by the exact value, is shown as a function of $D$. The analytical results of Ref. [7] in the infinite volume limit were used to draw the curve.

Fig.3: The motion of the pair of complex conjugate roots near the critical value for $n$ between 6 and 13. A $[8,8]$ Padé approximant was used for $D=3$.

Fig.4: The motion of all the roots for $n$ between 17 and 60 with a $[8,8]$ Padé approximant and $D=3$.

Fig.5 Distribution of the values of $\gamma$ obtained from Padé approximants with $L+$ $M+1 \leq 30$ and $D=3$.

\section{TABLE CAPTIONS}

Table 1: The coefficients of the high-temperature expansion of the susceptibility up to order 20 for the hierarchical model with $D=3$ and $2^{n}$ sites with $n=10,20$, 30 and 40. The last column is for the nearest neighbor model on a simple cubic lattice (see Ref. [10]). The temperature of the nearest neighbor model has been rescaled in such way that the first coefficients of the two last column coincide.

Table 2: The coefficients of the high-temperature expansion of the susceptibility up to order 30 for the hierarchical model with $D=3$ and 4 with $2^{60}$ sites.

Table 3: The difference between the exact and approximate values of the first 30 coefficients divided by the exact value, for the hierarchical model with $D=3$ and $2^{n}$ sites with $n=40$ and 60 . The last column is the same quantity for the nearest neighbor model on a simple cubic lattice.

Table 4: The values of $\beta_{c}$ and $-\gamma$ (between parenthesis) for $D=3$ obtained from $[j+k, j]$ Padé approximants, for $1 \leq j \leq 14$ and $k= \pm 1$ and 0 . A star indicates 
two very nearby poles.

Table 5: Same quantities as in Table 4 but for $D=4$. 


\begin{tabular}{|c|c|c|c|c|c|}
\hline \multicolumn{5}{|c|}{$b_{m, n}$ Hierarchical Model $D=3$} & \multirow{2}{*}{$\begin{array}{c}b_{m}^{s c}\left(\frac{b_{1}^{H M}}{b_{1}^{s c}}\right)^{m} \\
\text { Simple } \\
\text { Cubic }\end{array}$} \\
\hline $\mathrm{m} \backslash \mathrm{n}$ & 10 & 20 & 30 & 40 & \\
\hline 1 & 1.2258497 & 1.2424374 & 1.2426008 & 1.2426024 & 1.2426024 \\
\hline 2 & 1.2389539 & 1.2798863 & 1.2802922 & 1.2802962 & 1.2867173 \\
\hline 3 & 1.1820851 & 1.2490897 & 1.2497599 & 1.2497665 & 1.3146331 \\
\hline 4 & 1.0785253 & 1.1703159 & 1.1712435 & 1.1712527 & 1.2987587 \\
\hline 5 & 0.9628730 & 1.0760627 & 1.0772198 & 1.0772312 & 1.2804051 \\
\hline 6 & 0.8452212 & 0.9755497 & 0.9768990 & 0.9769123 & 1.2429628 \\
\hline 7 & 0.7316955 & 0.8746523 & 0.8761526 & 0.8761674 & 1.2051755 \\
\hline 8 & 0.6274001 & 0.7785983 & 0.7802084 & 0.7802243 & 1.1594697 \\
\hline 9 & 0.5339023 & 0.6893634 & 0.6910447 & 0.6910612 & 1.1146199 \\
\hline 10 & 0.4510918 & 0.6073781 & 0.6090960 & 0.6091130 & 1.0663937 \\
\hline 11 & 0.3787313 & 0.5329771 & 0.5347017 & 0.5347187 & 1.0196561 \\
\hline 12 & 0.3163770 & 0.4662812 & 0.4679872 & 0.4680040 & 0.9718193 \\
\hline 13 & 0.2631594 & 0.4069559 & 0.4086227 & 0.4086391 & 0.9258158 \\
\hline 14 & 0.2179946 & 0.3543998 & 0.3560112 & 0.3560270 & 0.8799407 \\
\hline 15 & 0.1798494 & 0.3079961 & 0.3095398 & 0.3095551 & 0.8360504 \\
\hline 16 & 0.1478169 & 0.2671874 & 0.2686545 & 0.2686690 & 0.7929596 \\
\hline 17 & 0.1210802 & 0.2314434 & 0.2328281 & 0.2328417 & 0.7518815 \\
\hline 18 & 0.0988810 & 0.2002368 & 0.2015355 & 0.2015483 & \\
\hline 19 & 0.0805232 & 0.1730514 & 0.1742628 & 0.1742747 & \\
\hline 20 & 0.0653886 & 0.1494050 & 0.1505292 & 0.1505403 & \\
\hline
\end{tabular}

Table 1 